\title{
Fondation APICIL - Agir ensemble contre la douleur www.fondation-apicil.org
}

\section{Nous soutenons vos projets \\ Vous pouvez proposer un projet à la Fondation APICIL, \\ en téléchargeant un dossier de demande de subvention sur le site internet : \\ www.fondation-apicil.org}

La fondation APICIL soutient des projets d'intérêt général visant à prévenir et à soulager la douleur, physique et psychique, à tous les âges de la vie.

Elle s'engage aux côtés de ceux qui œuvrent pour soulager la douleur et la souffrance. Elle accompagne, soutient, agit aux côtés des organismes publics ou privés du monde médical et des associations, pour que les pratiques évoluent, et que la douleur soit atténuée.

La fondation APICIL a été créée par le groupe APICIL, groupe de protection sociale lyonnais, engagé depuis de nombreuses années dans un mécénat social, significatif et diversifié dans le domaine du handicap, de la santé, du grand âge, du deuil et de l'emploi.

Reconnue d'utilité publique, en 2004, la fondation APICIL est dotée de neuf millions d'euros et dispose, chaque année, d'un budget opérationnel conséquent qui lui permet d'accompagner de nombreux projets en France métropolitaine. Présidée par Michel Angé et dirigée par Nathalie Aulnette, elle est également encadrée par un conseil d'administration et un conseil scientifique engagé, composé de personnalités du monde médical qui mettent à disposition leurs compétences afin d'orienter le choix des projets soutenus.

"Les connaissances scientifiques, les moyens financiers et humains sont réunis pour permettre aux soignants et aux patients de disposer de solutions nouvelles et complémentaires pour soulager la douleur et la souffrance. »

\section{Nathalie Aulnette, directrice de la fondation APICIL}

Depuis sa création, la fondation APICIL a accompagné plus de 90 projets visant à prévenir et à soulager la douleur, à travers des actions variées :

- recherche ;

- formation ;

- information ;

- matériel ;

- prix et bourses ;

- pratiques nouvelles.

\section{Exemple de projet}

Parmi les pratiques innovantes pour soulager la douleur, l'hypnoanalgésie a été particulièrement encouragée par la fondation APICIL. Elle a notamment permis la formation du service de pédiatrie de l'hôpital de Villefranche (69). Les évaluations montrent une diminution de la douleur lors des soins ou en postopératoire, une diminution du stress, des cris et des pleurs dans le service.

Lorsque Paul (six ans) arrive à l'hôpital de Villefranchesur-Saône pour subir une prise de sang, il n'est pas rassuré. Les infirmières l'informent, le rassurent et l'interrogent sur ses centres d'intérêt, sa réponse fuse: "le football». Aussitôt, l'infirmière, formée à l'hypnoanalgésie, lui propose une partie de football virtuelle, tandis que la seconde infirmière procède aux soins (Fig. 1).

En quelques secondes, le visage de l'enfant passe de l'inquiétude à l'apaisement. Les soins sont effectués dans un climat de détente, parents, soignants et soigné sont satisfaits.

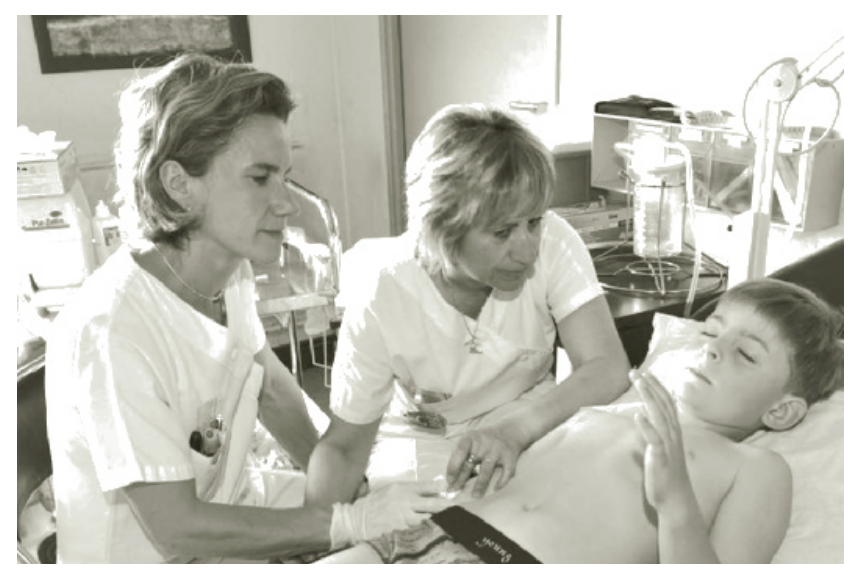

Fig. 1 Prise de sang sous hypoanalgésie

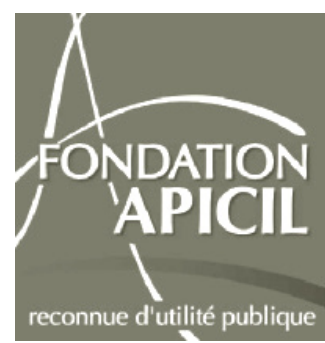

